\title{
Rotational Symmetry of Solutions of Some Nonlinear Problems in Statistical Mechanics and in Geometry
}

\author{
Sagun Chanillo, Michael Kiessling \\ Department of Mathematics, Rutgers University, New Brunswick, NJ 08903, USA
}

Received: 26 February 1993/in revised form: 21 June 1993

\begin{abstract}
The method of moving planes is used to establish a weak set of conditions under which the nonlinear equation $-\Delta u(x)=V(|x|) e^{u(x)}, x \in \mathbb{R}^{2}$ admits only rotationally symmetric solutions. Additional structural invariance properties of the equation then yield another set of conditions which are not originally covered by the moving plane technique. For instance, nonmonotonic $V$ can be accommodated. Results for $-\Delta u(y)=V(y) e^{u(y)}-c$, with $y \in S^{2}$, are obtained as well. A nontrivial example of broken symmetry is also constructed. These equations arise in the context of extremization problems, but no extremization arguments are employed. This is of some interest in cases where the extremizing problem is neither manifestly convex nor monotone under symmetric decreasing rearrangements. The results answer in part some conjectures raised in the literature. Applications to logarithmically interacting particle systems and geometry are emphasized.
\end{abstract}

\section{Introduction and Main Results}

The present paper is concerned with spatial symmetry and symmetry breaking of solutions of certain nonlinear field equations in two-dimensional domains without boundary. The presence of (symmetric) boundaries is known to catalyze symmetry, as well as its breaking, in many circumstances. In the absence of boundaries, on the contrary, such features reflect intrinsic properties of the system under study. Various approaches to this type of problem exist in the literature, the applicability of which depends in part on the specific problem under consideration. We will be using here the method of moving planes [A, GNN1, GNN2, Li, LN1, LN2, LN3, $\mathrm{CheL}$ ], which is based on the maximum principle and allows us to prove conditions under which any solution of our equations is symmetric.

We recall that so far, the moving plane method has shown the absence of symmetry breaking, under mild conditions, for positive solutions of the conformally invariant equations

$$
\Delta u+u^{p}=0 ; \quad x \in \mathbb{R}^{n}
$$


with

$$
p=\frac{n+2}{n-2}
$$

and $n \geqq 3$, see [GNN1, CGS], which in the case $n=4$ is known to generate solutions in a regular gauge of the classical Euclidean Yang-Mills equations, via 't Hooft's Ansatz [Wilc]. All positive solutions are known and given by

$$
\begin{aligned}
{\left[u_{\lambda}^{(n)}\left(x ; x_{0}\right)\right]^{\frac{n+2}{n-2}} } & =C(n, \lambda)\left(1+\lambda^{-2}\left|x-x_{0}\right|^{2}\right)^{-\frac{n+2}{2}}, \\
C(n, \lambda) & =\left(n[n-2] \lambda^{-2}\right)^{\frac{n+2}{4}}
\end{aligned}
$$

with $x_{0} \in \mathbb{R}^{n}$ the arbitrary center of symmetry, and $\lambda \in \mathbb{R}^{+}$the arbitrary length scale. Whence the well-known one-instanton solutions are all Yang-Mills solutions that $(1.1 \mathrm{a} / \mathrm{b})$ generates. For $n=3,(1.1 \mathrm{a} / \mathrm{b})$ is known as Plummer's model in the theory of galactic structure; see [Al] for some rigorous results. The corresponding result on $\mathbb{R}^{2}$ was supplied by Chen and $\mathrm{Li}[\mathrm{CheL}]$. The counterpart of $(1.1 \mathrm{a} / \mathrm{b})$ in $\mathbb{R}^{2}$ is Liouville's equation

$$
\Delta u(x)+e^{u(x)}=0 ; \quad x \in \mathbb{R}^{2},
$$

which under the vital condition of finite total generating mass,

$$
\int_{\mathbb{R}^{2}} e^{u} d^{2} x<\infty
$$

admits only solutions which are radial about some point (Theorem. 1 of [CheL]). All such radial solutions are known and given by

$$
\exp \left[u_{\lambda}^{(2)}\left(x ; x_{0}\right)\right]=8 \lambda^{-2}\left(1+\lambda^{-2}\left|x-x_{0}\right|^{2}\right)^{-2} .
$$

For the sake of completeness, we mention that the results of [GNN1, CGS] have a geometric analog on $S^{n}, n \geqq 3$, proved by Obata [Ob]; the results of [CheL] similarly have a geometric analog on $S^{2}$; see also [Sch].

It should be noted in the above that the method of moving planes itself is a tool to prove symmetry of solutions under the assumption that they exist. However, once symmetry is established, existence follows in general readily by a standard ODE argument. Furthermore, this PDE method does not discriminate between solutions which have the additional virtue of being optimizers in some extremal problem and those which are not. This appears as a disadvantage, but may also turn into an advantage, as one is not always only interested in the optimizers. All the above solutions $(1.1 \mathrm{c} / \mathrm{d})$ and $(1.2 \mathrm{c})$ in fact are extremizers in conformally invariant inequalities; see, for instance, [Lie, CaL1] for (1.1c), [CaL2] for (1.2c), [On] for the counterpart of (1.2c) on $S^{2}$, [Be] for $S^{4}$, [Br] for $S^{4}$ and $S^{6}$, [CaL2] for $S^{n}$; and [OPS] for the more general compact closed surfaces. As such their symmetry follows from symmetric decreasing rearrangement arguments. These extremal problems leave open the question of existence of other solutions to $(1.1 \mathrm{a} / \mathrm{b})$ and $(1.2 \mathrm{a} / \mathrm{b})$, which may be of interest in some other context that does not require extremal properties. The moving plane technique tells us in these cases there are no other "natural" solutions.

The above equations are of the type $\Delta u+F(u)=0$. Results in all $\mathbb{R}^{n}$ exist also for fully nonlinear equations $F(u,|\nabla u|$, det $\operatorname{Hess}(u), \ldots)=0$, see $[\mathrm{GNN} 1]$, 
Theorem 4. For equations with explicit $x$-dependence, like $\Delta u+G(u ; x)=0$, results for finite balls are also given in [GNN1], Theorem 1'. The problem in all of $\mathbb{R}^{n}$ is more delicate as the lack of compactness has to be compensated by apriori information about asymptotic behavior. For $n \geqq 3$ the problem was overcome in [Li] for quite general fully nonlinear equations $F(x, u,|\nabla u|$ , det $\operatorname{Hess}(u), \ldots)=0$, under a-priori assumptions on asymptotic decay properties of $F . \mathrm{Li}$ and $\mathrm{Ni}[\mathrm{LN} 1, \mathrm{LN} 2]$ treat more special equations $\Delta u+V(x) g(u)=0, \quad x \in \mathbb{R}^{n}, \quad n \geqq 3$, with certain asymptotically power law decaying $V$ and for $g=\exp$ and $g(\cdot)=(\cdot)^{p}$; these cases are generalized in [LN3] but still restricted to $n>2$. The corresponding problem for $n=2$ is critical and not covered by the results of [Li, LN1, LN2, LN3].

In this paper we adapt the method of moving planes to study elliptic scalar second order PDEs of the form

$$
-\Delta u(x)=V(x) e^{u(x)}
$$

with $x \in \mathbb{R}^{2}$. We construct an almost minimal set of conditions on $V$ under which the moving plane method allows us to conclude that any solution of $\left(1.3^{p}\right)$ is rotational symmetric and radial decreasing. Liouville's equation will be included as a special case. We remark that in a specific situation additional a-priori information on $V$ might be available. Some of our conditions may then be improvable. We give two examples in our section on applications. Rather than trying to improve further some moving-plane estimates at the expense of more and more specialized $V$, we find it more interesting to merely use the structural invariance of $\left(1.3^{p}\right)$ under Kelvin transformations to obtain symmetry theorems under conditions on $V$ which are not originally covered by the moving plane method. Using stereographic projection techniques, we also study the related problem,

$$
\begin{aligned}
-\Delta_{S} u(y) & =V(y) e^{u(y)}-c, \\
4 \pi c & =\int_{S^{2}} V(y) e^{u(y)} d \mu
\end{aligned}
$$

with $y \in S^{2}, d \mu$ the uniform measure on $S^{2}$ w.r.t. the standard metric, $c$ a positive constant, and obtain conditions on $V$ under which any solution of $\left(1.3^{s}\right)$ is rotational symmetric. In $\left(1.3^{s}\right), \Delta_{S}$ is the Laplace Beltrami operator w.r.t. the standard metric. Equations $\left(1.3^{p}\right)$ and $\left(1.3^{s}\right)$ arise in geometry and various branches of physics, for instance fluid vortex distributions in decaying turbulence. Applications of our results will be discussed in Sect. 7 .

We remark that in the following we understand $V$ to be continuous and $u$ to be a classical solution. By a simple limiting argument based on super- and subsolutions one can extend our results to discontinuous $V$. We also prove some results for $\left(1.3^{p}\right)$ which are valid for more general nonradial $V$.

We now state our symmetry theorems for Eqs. $\left(1.3^{p}\right)$ and $\left(1.3^{s}\right)$. In order to apply the method of moving planes to $\left(1.3^{p}\right)$, we will supplement $\left(1.3^{p}\right)$ by some or all of the following hypotheses:

$$
\begin{gathered}
V \in L^{\infty}\left(\mathbb{R}^{2}\right) \\
V\left(x^{\prime}\right) \leqq V(x) ; \quad\left|x^{\prime}\right|>|x|,
\end{gathered}
$$


where $|x|=\langle x, x\rangle^{1 / 2} \equiv r$ is the Euclidean norm of $x \in \mathbb{R}^{2}$,

$$
\begin{gathered}
\int_{\mathbb{R}^{2}}|V(x)| e^{u(x)} d^{2} x<\infty, \\
\int_{\mathbb{R}^{2}} V(x) e^{u(x)} d^{2} x>6 \pi, \\
u^{+}(x)=\max \{u(x), 0\} \in L^{1}\left(\mathbb{R}^{2}, d^{2} x\right) .
\end{gathered}
$$

We will always explicitly state which of the above hypotheses are needed. Our moving plane result is

Theorem P1. Under the hypotheses (HP1)-(HP5), any solution of $\left(1.3^{p}\right)$ is necessarily radial symmetric and decreasing.

Structural invariance of $\left(1.3^{p}\right)$ under Kelvin transformations allows us to accommodate the following set of hypotheses:

$$
V(x)=|x|^{m-4} U(x)
$$

with $2 \pi m=1 . h . s .(H P 4)$,

$$
\begin{gathered}
U(x) \in L^{\infty}, \\
U\left(x^{\prime}\right) \geqq U(x) ; \quad\left|x^{\prime}\right|>|x|, \\
\max \{v(x), 0\} \in L^{1}\left(\mathbb{R}^{2},|x|^{-4} d^{2} x\right), \\
v(x)=u(x)+m \ln |x| .
\end{gathered}
$$

We will explicitly state when we assume $V$ to satisfy $\left(\mathrm{HP} 1^{*}\right),\left(\mathrm{HP} 2^{*}\right),\left(\mathrm{HP} 5^{*}\right)$.

Theorem P2. Under the hypotheses (HP1*), (HP2*), (HP3), (HP4), (HP5*) on $V(x)$, any solution of $\left(1.3^{p}\right)$ is necessarily radial symmetric. It is asymptotically decreasing for large $|x|$, but not necessarily decreasing a.e.

Certain non-monotonic $V(x)$ which are not in $L^{\infty}$ are covered by Theorem P2. The control over radial decrease is lost in the transformations.

A statement very close in appearance to Theorem P1 can be made for $\left(1.3^{s}\right)$ and is obtained from Theorem P1 by stereographic projection techniques. We supplement $\left(1.3^{s}\right)$ by some or all of the following hypotheses:

$$
\begin{gathered}
V \in L_{+}^{\infty}\left(S^{2}\right), \\
V(y) \leqq V\left(y^{\prime}\right) ; \quad\langle\mathbf{n}, y\rangle>\left\langle\mathbf{n}, y^{\prime}\right\rangle,
\end{gathered}
$$

for some fixed $\mathbf{n} \in S^{2}$,

$$
\begin{gathered}
\int_{S^{2}} V(y) e^{u(y)} d \mu \leqq 8 \pi, \\
\int_{S^{2}} V(y) e^{u(y)} d \mu>6 \pi, \\
u^{+}(y) \in L^{1}\left(S^{2} ;[1-\langle\mathbf{n}, y\rangle]^{-2} d \mu\right) .
\end{gathered}
$$

We will always explicitly state which of the above hypotheses are needed.

Theorem S1. Under the hypotheses (HS1)-(HS5), any solution of $\left(1.3^{s}\right)$ is necessarily rotational invariant around $\mathbf{n}$. 
One can also show (see $[\mathrm{CK}]$ ) that under (HS1)-(HS5) the solutions of $\left(1.3^{s}\right)$ are monotone decreasing as function of $\langle\mathbf{n}, y\rangle$, but this is lost in the mapping from Theorem P1.

Interestingly, Theorem S1 does not include all the information of Theorem P1. Notice the differences (HP1) vs. (HS1), and (HP3) vs. (HS3). Theorems P1 and P2 can be fully combined into a single Theorem for $\left(1.3^{s}\right)$. We need the hypotheses

$$
V(y)=(1-\langle\mathbf{n}, y\rangle)^{c-2} U(y)
$$

for some fixed $\mathbf{n} \in S^{2}$,

$$
\begin{gathered}
U(y) \in L^{\infty}\left(S^{2}\right), \\
U(y) \leqq U\left(y^{\prime}\right) ;\langle\mathbf{n}, y\rangle>\left\langle\mathbf{n}, y^{\prime}\right\rangle, \\
\int_{S^{2}}|V(y)| e^{u(y)} d \mu<\infty, \\
\max \{v(y), 0\} \in L^{1}\left(S^{2} ;[1-\langle\mathbf{n}, y\rangle]^{-2} d \mu\right), \\
v(y)=u(y)+c \ln \left(\frac{1}{2}[1-\langle\mathbf{n}, y\rangle]\right) .
\end{gathered}
$$

We will explicitly state when we assume $V$ to satisfy (HS1*), (HS2*), (HS5*).

Theorem S2. Under the hypotheses (HS1*)-(HS3*), (HS4), (HS5*), any solution of $\left(1.3^{s}\right)$ is necessarily rotational invariant around $\mathbf{n}$.

Before we start verifying our claims, several remarks are in order. As far as our list of hypotheses (HP1)-(HP5) is concerned, we hope to convey to the reader that it is close to the minimal set of hypotheses which would be needed to prove statements like Theorem P1 with the moving plane technique, but not completely optimal. As far as the method of moving planes itself is concerned, we see that it falls short of capturing all reasonable conditions on $V$ such that $\left(1.3^{p}\right)$ has only rotationally symmetric solutions. This becomes plain through Theorem P2. We will also see that some of our hypotheses are intrinsically sharp.

(R1): Hypothesis (HP1) is satisfied in some applications that we have in mind, but does not seem to be vital given the other hypotheses hold. The emphasis here is on keeping all other hypotheses. We will see below that the interplay of (HP1) and (HP2) is crucial. Given (HP2)-(HP5) hold, one may try to relax (HP1) and lump it together with (HP3) to $V \in L^{p}, e^{u} \in L^{p^{\prime}}, p^{-1}+p^{-1}=1$, as in the blow-up study of [BM]. A similar remark applies to (HS1). A model with $V \sim r^{-\alpha}$ at the origin and decreasing in $r$ occurs in screening problems, for which radial solutions have been studied in [GP]. Our Theorem P2 includes some such $V$ but is not directly obtained by moving plane arguments. A weakening of (HP1), and (HS1), is therefore desirable.

(R2): The non-increase hypothesis (HP2) is needed for some reflection estimates which are vital for the moving plane technique; cf. [GNN]. (HP2) implies also rotational symmetry of $V$, which is a trivial sine qua non to find radial solutions of $\left(1.3^{p}\right)$, and this holds true for (HS2) regarding $\left(1.3^{s}\right)$.

(R3): The counterpart of (HP2) on $S^{2}$, i.e., (HS2), demands monotonicity along some $\mathbf{n} \in S^{2}$. It is thus suggestive that also for $\left(1.3^{p}\right)$ the important property is not 
(HP2) but just monotonicity, at least if boundedness of $V$ is kept. This, however, may be false in general. The full analog of Theorem S1 is a special case of our Theorem P2. Nevertheless, it covers nonmonotonic $V$ and shows that (HP2) is a technical restriction which comes from the moving plane method, not an intrinsic requirement.

(R4): If, however, we relax (HP1) and admit unbounded $V$, then (HP2) seems to be vital in the following sense. If besides dropping (HP1) we also replace (HP2) by the weaker requirement that $V$ is radial, but otherwise keep all further hypotheses, we can construct counterexamples. The verification of this claim is relegated to Sect. 6, where for power law increasing $V$ we construct nonradial $C^{\infty}$ solutions of $\left(1.3^{p}\right)$ with finite generating mass $>8 \pi$, with $u^{+}$compactly supported on a bounded simply connected domain. Superficially this seems to be in conflict with Theorem P2. We will see, however, that the hypotheses of Theorem P2 imply that $m \leqq 4$.

We remark that counterexamples to rotational symmetry for bounded increasing $V$ for simpler (linear) equations for $u$ in a finite ball are given in [GNN1].

(R5): (HP3) is intrinsically vital in the sense that dropping (HP3) but keeping all other hypotheses allows us to construct examples of $C^{\infty}\left(\mathbb{R}^{2}\right)$ solutions of $\left(1.3^{p}\right)$ with broken symmetry but infinite generating mass. To see that (HP3) is not just a technical restriction, we give a nontrivial explicit counterexample to radial symmetry in the special case of $\left(1.3^{p}\right)$ with a trivial weight $V \equiv 1$, i.e., Liouville's equation (1.2a). We note that [CheL] need (1.2b), i.e., our (HP3), to prove their symmetry theorem for (1.2a).

Indeed, for any $\mathbf{v}$ and $\mathbf{v}^{\prime}$ in $S^{1} \subset \mathbb{R}^{2}$ with $\left\langle\mathbf{v}, \mathbf{v}^{\prime}\right\rangle=0$, and any $\zeta \in \mathbb{R}, \kappa \in \mathbb{R}^{+}$and $x_{0} \in \mathbb{R}^{2}$, the function $U_{\zeta, \kappa}\left(x ; x_{0}\right)$ given by

$$
\begin{aligned}
& \exp \left[U_{\zeta, \kappa}\left(x ; x_{0}\right)\right]= \\
& 2 \kappa^{2}\left\{\cosh \zeta \cosh \left\langle\mathbf{v}, \kappa\left(x-x_{0}\right)\right\rangle+\sinh \zeta \cos \left\langle\mathbf{v}^{\prime}, \kappa\left(x-x_{0}\right)\right\rangle\right\}^{-2}
\end{aligned}
$$

is a periodic $C^{\infty}$ solution of $\left(1.3^{p}\right)$ for the constant weight $V \equiv 1$. Solution (1.4) has infinite generating mass and therefore it violates (HP3). Note that $\exp \left(U_{\zeta, \kappa}\right) \in L^{\infty}$. It is of course a special case of Liouville's general solution. It was discovered independently by Stuart [St] and by Schmid-Burgk [SB]. However,

$$
\left\|U_{\zeta, \kappa}^{+}\right\|_{\infty}=2|\zeta|+\ln 2 \kappa^{2}
$$

which is $>0$ for $\kappa>e^{-|\zeta|} / \sqrt{2}$, and thus (1.4) also violates (HP5). This is easily corrected by defining $u_{\zeta, \kappa}=U_{\zeta, \kappa}-\left\|U_{\zeta, \kappa}^{+}\right\|_{\infty}$ and $V_{\zeta, \kappa}=\exp \left\|U_{\zeta, \kappa}^{+}\right\|_{\infty}$. Then $u_{\zeta, \kappa}$ is a nonradial solution of $\left(1.3^{p}\right)$ for this particular $V$; it satisfies all hypotheses but (HP3).

(R6): If $V$ is not everywhere positive, our condition (HP3) is somewhat stronger than demanding finite total generating mass. At present we do not know whether (HP3) remains that vital in case of a nontrivial $V$ which takes negative values on a set of finite measure, or whether it can be relaxed to some improper sense of $\int V e^{u}<\infty$. In fact, a definition of an improper integral may be unnecessary by demanding instead a uniform upper bound on $\int_{\Lambda} V e^{u} d^{2} x$ on nested compact subsets $\Lambda \subset \mathbb{R}^{2}$. This is an open problem, however. We add that (HP3) rules out also some $u$ which are solutions only in the distributional sense.

(R7): Condition (HP4) enters through the moving-plane argument. Hypothesis (HP4) is sharp in the moving plane arguments because we do not make any a-priori 
assumptions on asymptotic behavior of $V$. It is an interesting open question whether (HP4) is sharp also in the following PDE sense: Is it possible to find a $V(x)$ and a corresponding nonradial solution $u$ of $\left(1.3^{p}\right)$ which satisfy (HP1)-(HP3) and (HP5), but (HP4) is violated?

In Proposition 1 (Sect. 5) we state a sufficiency condition for $V$ which implies that any solution of $\left(1.3^{p}\right)$ satisfies (HP4). In these cases (HP4) is automatically sharp. If the sufficiency condition is not satisfied, it does not seem a-priori obvious that for given $V$ there will be a solution of $\left(1.3^{p}\right)$ satisfying (HP4). In a forthcoming work we address this problem of whether (HP4) is intrinsically sharp with a very different technique, see [CK].

On the other hand, in more specialized cases where some asymptotic decay to zero of $V$ is known a-priori, our (HP4) can be improved. See Sect. 7.

(R8): Inequality (HS3) enters through the stereographic projection; see Sect. 4. We will see in Sect. 6 that well behaved but not rotational symmetric solutions of $\left(1.3^{s}\right)$ which violate (HS3) are likely to exist. (HS3) is stronger than (HP3); nevertheless it turns out that such a bound is automatically satisfied in $\mathbb{R}^{2}$ under the conditions expressed in Theorem $\mathrm{P} 1$, and on $S^{2}$ under the conditions of Theorem S2. See Sect. 4.

(R9) Condition (HP5) is again vital given we do not know anything more than (HP1)-(HP4) and the fact that $u$ solves $\left(1.3^{p}\right)$. It enters technically through Lemma 1, which we need for the proof of Theorem P1. The proof of Lemma 1 is based on an a-priori $L^{\infty}$ estimate for $u^{+}$as given in [BM], Theorem 2. In [BM], instead of our (HP1), (HP3) the conditions $V \in L^{p}$, $e^{u} \in L^{p^{\prime}}, p \in(1, \infty]$, with $p$ and $p^{\prime}$ conjugate exponents, are employed, which for $p=\infty$ [our (HP1)] yields $e^{u} \in L^{1}$, in which case our (HP5) follows; see [BM]. Clearly, for $p=\infty$ the [BM] condition is somewhat stronger than our (HP1), (HP3). One may want to try to generalize the [BM] argument to get an $L^{\infty}$ result for $u^{+}$on basis of (HP3), but compactly supported $V$ provide us with counterexamples. Notice that $u$ is harmonic on $\mathbb{R}^{2} \backslash \operatorname{supp}(V)$, so it is easy to construct $u$ which tend to $+\infty$ as $|x|$ becomes large in certain directions of space. Physically these asymptotically harmonic functions correspond to external sources at infinity, which generically destroy the rotational symmetry. As we are interested in system-intrinsic results, we have to control the asymptotic behavior of $u^{+}$. Our condition (HP5) is relatively mild, but in fact sufficiently strong to rule our sources at infinity.

(R10): The various hypotheses (HS5) are analogs of (HP5) on $S^{2}$. The strange factors enter through the stereographic projection.

(R11): If a little more is known about $V$, for instance $V$ is bounded away from 0 , then we can drop (HP5). This is clear as $V \geqq c>0$ together with (HP1) and (HP3) imply that $e^{u} \in L^{1}$, and we are back to the [BM] estimate that we need in Lemma 1.

This ends our remarks on our system of hypotheses. It remains to outline the structure of the rest of the paper. In the next section we prepare the proof of our Theorem P1, which itself is presented in Sect. 3. In Sect. 4 we prove Theorems P2, S1 and S2. Section 5 offers a sufficiency condition on $V$ implying that any solution of $\left(1.3^{p}\right)$ (so it exists) necessarily satisfies (HP4). In Sect. 6 we construct a class of nonradial solutions of $\left(1.3^{p}\right)$ for radial $V$ satisfying all constraints but (HP2) and (HP1). In Sect. 7, some applications to physics and geometry are discussed. For vortex systems we expect our results signal a symmetry breaking phase transition. 


\section{Preliminaries}

For the proof of Theorem P1 we need

Lemma 1. Let $u$ be a solution of $\left(1.3^{p}\right), x \in \mathbb{R}^{2}$. Assume (HP1), (HP3), (HP5) hold. Assume further that $\int_{\mathbb{R}^{2}} V(x) e^{u} d^{2} x>0$. Then

$$
\lim _{|x| \rightarrow \infty} \frac{u(x)}{\ln |x|}=-\frac{1}{2 \pi} \int_{\mathbb{R}^{2}} V(x) e^{u(x)} d^{2} x .
$$

Proof. Let

$$
\Psi(x)=\frac{1}{2 \pi} \int_{\mathbb{R}^{2}}(\ln |x-y|-\ln |y|) V(y) e^{u(y)} d^{2} y .
$$

Then,

$$
\Delta \Psi(x)=V(x) e^{u(x)} .
$$

Since $u$ satisfies $\left(1.3^{p}\right)$ and $\Psi$ satisfies (2.3), the function $h(x)=u(x)+\Psi(x)$ is harmonic. By hypothesis (HP1), $V \in L^{\infty}\left(\mathbb{R}^{2}\right)$. By (HP3), $V e^{u} \in L^{1}\left(\mathbb{R}^{2}\right)$. By (HP5), $u^{+} \in L^{1}\left(\mathbb{R}^{2}\right)$. Thus by examining Theorem 2 of [BM] or by Corollary 3.3, p. 430 of [ChaL], we conclude that $\left\|u^{+}\right\|_{\infty}<\infty$. Moreover, by the definition of $\Psi$,

$$
\lim _{|x| \rightarrow \infty} \frac{\Psi(x)}{\ln |x|}=\frac{1}{2 \pi} \int_{\mathbb{R}^{2}} V(x) e^{u(x)} d^{2} x .
$$

This implies that the harmonic function $h$ is bounded by

$$
h(x) \leqq u^{+}(x)+\Psi(x) \leqq C+C^{\prime}[\ln (|x|+10)] .
$$

Thus $h(x) \equiv C^{\prime \prime}$. Therefore

$$
\lim _{|x| \rightarrow \infty} \frac{u(x)}{\ln |x|}=-\lim _{|x| \rightarrow \infty} \frac{\Psi(x)}{\ln |x|},
$$

which proves Lemma 1. Q.E.D.

Corollary 1. Assume u satisfies $\left(1.3^{p}\right)$ with (HP1), (HP3)-(HP5). Then for some $\varepsilon>0$ we have uniformly

$$
e^{u(x)} \leqq C|x|^{-3-\varepsilon}
$$

as $|x| \rightarrow \infty$.

Proof. By (HP4), for some $\delta>0$,

$$
\int_{\mathbb{R}^{2}} V(x) e^{u(x)} d^{2} x \geqq 6 \pi+\delta .
$$

By Lemma 1,

$$
\lim _{|x| \rightarrow \infty} \frac{u(x)}{\ln |x|}=-\frac{1}{2 \pi} \int_{\mathbb{R}^{2}} V(x) e^{u(x)} d^{2} x \leqq-\frac{6 \pi+\delta}{2 \pi}=-3-\varepsilon .
$$

Thus

$$
e^{u(x)}=|x|^{u(x) / \ln |x|} \leqq C|x|^{-3-\varepsilon},
$$

provided $|x| \geqq r_{0}$ for some $r_{0}$. Q.E.D. 
We continue preparing the proof of Theorem P1. Note that by Lemma 1 , if $\int_{\mathbb{R}^{2}} V(x) e^{u} d^{2} x>0$, then $u(x) \rightarrow-\infty$ as $|x| \rightarrow \infty$ uniformly. Thus it is possible to talk of a global maximum for $u$. We will now show that without loss of generality we can arrange coordinates so that this global maximum is at the point $(-4,0)$. We shall moreover see that these coordinate transformations leave (HP1, 3, 4, 5) invariant.

First note that by a coordinate rotation $\sigma$ we can arrange coordinates so that the function $u \circ \sigma=u_{\sigma}$ has its maximum at the point $(-a, 0)$, with $a \geqq 0$. We have to distinguish two cases.

Case 1: Let $a>0$. In this case we scale coordinates to a new system via $z=4 x / a$. We set $\mu=4 / a$. Now notice that $u_{\sigma}$ satisfies the equation $-\Delta u_{\sigma}(x)=V(\sigma x) e^{u_{\sigma}(x)}$. We will henceforth denote $V(\sigma x)$ by $V_{\sigma}(x)$ (we do not use (HP2) here). Thus $-\Delta u_{\sigma}(z / \mu)=V_{\sigma}(z / \mu) e^{u_{\sigma}(z / \mu)}$. Denoting the function $u_{\sigma}(z / \mu)$ by $\bar{u}(z)$ and $\mu^{-2} V_{\sigma}(z / \mu)$ by $\bar{V}(z)$, we see that $\bar{u}$ satisfies the equation $-\bar{\Delta} \bar{u}(z)=\bar{V}(z) e^{\bar{u}(z)}$, where $\bar{\Delta}$ denotes the scaled Laplace operator. It is easy to check that, if the pair $u$ and $V$ satisfies (HP1, 3, 4, 5), then so does the pair $\bar{u}$ and $\bar{V}$. Thus, if $a>0$, we can always assume without loss of generality that $a=4$. The proof of our Theorem P1 will eventually show us that this case cannot arise unless $V$ is constant, so that $a>0$ does not arise unless $V$ is constant.

Case 2: Let $a=0$. Here we translate the origin to $(4,0)$. Set $\bar{u}(x)=u\left(x_{1}+4, x_{2}\right)$, with $x=\left(x_{1}, x_{2}\right)$. Let $\bar{V}(x)=V\left(x_{1}+4, x_{2}\right)$. Then from $\left(1.3^{p}\right), \bar{u}(x)$ satisfies $-\Delta \bar{u}(x)=\bar{V}(x) e^{\bar{u}(x)}$ in $\mathbb{R}^{2}$. Note that, if the pair $u$ and $V$ satisfies $(\mathrm{HP} 1,3,4,5)$, then so does the pair $\bar{u}$ and $\bar{V}$.

Thus, if $u$ and $V$ satisfies (HP1, 3, 4, 5), we have converted our problem to a new pair $\bar{u}$ and $\bar{V}$, with $\bar{V}=\mu^{-2} V(\sigma x / \mu)$ in Case 1 , and with $\bar{V}=V\left(x_{1}+4, x_{2}\right)$ in Case 2. We shall now simply denote $\bar{u}$ by $u$ and thus our new equation is

$$
-\Delta u(x)=\bar{V}(x) e^{u(x)} \text {. }
$$

We introduce some notation. Let $\Sigma_{\lambda}=\left\{x: x=\left(x_{1}, x_{2}\right)\right.$ with $\left.x_{1}<\lambda\right\}$ denote a half space, and let $T_{\lambda}=\left\{x: x_{1}=\lambda\right\}$ be a line (a "plane"). For a given $x$, let $x_{\lambda}$ denote its reflection across $T_{\lambda}$, that is $x_{\lambda}=\left(2 \lambda-x_{1}, x_{2}\right)$. Next we prove

Lemma 2. Let $V$ satisfy (HP2). Then for $x_{1} \leqq \lambda \leqq-4$, and in particular for $x \in \Sigma_{\lambda}$, $\lambda \leqq-4, \bar{V}(x) \leqq \bar{V}\left(x_{\lambda}\right)$.

Proof. According to our construction, we have to consider two cases for $V$.

Case 1: In this case, $\bar{V}(x)=\mu^{-2} V(\sigma x / \mu)$, where $\sigma$ is a rotation. Since $V$ is radial symmetric as a consequence of (HP2), it follows that $\bar{V}(x)=\mu^{-2} V(x / \mu)$. Since $|x| \geqq\left|x_{\lambda}\right|$ for $x_{1} \leqq \lambda \leqq-4$, our claim follows from the fact that $V$ is decreasing by (HP2).

Case 2: In this case, $\bar{V}(x)=V\left(x_{1}+4, x_{2}\right)$, and thus $\bar{V}\left(x_{\lambda}\right)=V\left(2 \lambda-x_{1}+4, x_{2}\right)$. To verify our claim we now have to show that for $x_{1} \leqq \lambda \leqq-4$, we have $\left(x_{1}+4\right)^{2}+x_{2}^{2} \geqq\left(2 \lambda-x_{1}+4\right)^{2}+x_{2}^{2}$, which is evident. This concludes our proof of Lemma 2. Q.E.D.

Next we introduce $u_{\lambda}(x)=u\left(x_{\lambda}\right)$, then $v_{\lambda}(x)=u_{\lambda}(x)-u(x)$ and $g(x)=$ $\ln (|x|-1)$. We finally set $w_{\lambda}(x)=v_{\lambda}(x) / g(x)$. Notice that for $x \in \Sigma_{\lambda}, \lambda<-2$, the function $w_{\lambda}(x)$ is well defined. We see from (2.11) that

$$
-\Delta u_{\lambda}(x)=\bar{V}\left(x_{\lambda}\right) e^{u_{\lambda}(x)} \text {. }
$$


For $x \in \Sigma_{\lambda}, \lambda \leqq-4$, Lemma 2 applied to the identity above yields $\Delta u_{\lambda}(x)+$ $\bar{V}(x) e^{u_{\lambda}(x)} \leqq 0$. The inequality above coupled with $(2.11),(2.12)$ yields $v_{\lambda}(x)+$ $\bar{V}(x)\left(e^{u_{\lambda}(x)}-e^{u(x)}\right) \leqq 0$. By the mean value theorem there exists a $\psi(x)$ with values between $u_{\lambda}(x)$ and $u(x)$, such that $e^{u_{\lambda}(x)}-e^{u(x)}=v_{\lambda}(x) e^{\psi(x)}$. Thus $v_{\lambda}(x)$ satisfies the partial differential inequality

$$
\Delta v_{\lambda}(x)+\bar{V}(x) e^{\psi(x)} v_{\lambda}(x) \leqq 0 .
$$

We are now in the position to prove

Lemma 3. Under (HP1)-(HP5) there exists $R_{0}>0$ such that for $x_{0}$ a minimum point of $w_{\lambda}(x)$ in $\Sigma_{\lambda}, \lambda \leqq-4$, if $w_{\lambda}\left(x_{0}\right)<0$, then $\left|x_{0}\right|<R_{0}$.

Proof. First recall that by definition $w_{\lambda}(x)=v_{\lambda}(x) / g(x)$. Since $v_{\lambda}(x)$ satisfies the partial differential inequality (2.13), we see that $w_{\lambda}(x)$ satisfies the partial differential inequality

$$
\Delta w_{\lambda}(x)+2 \frac{\nabla g}{g} \cdot \nabla w_{\lambda}(x)+\left(\frac{\Delta g}{g}+\bar{V}(x) e^{\psi(x)}\right) w_{\lambda}(x) \leqq 0
$$

Next,

$$
\frac{\Delta g}{g}=-\left[|x|(|x|-1)^{2} \ln (|x|-1)\right]^{-1}
$$

We will now produce a contradiction, that is, assume that no such $R_{0}$ exists. Then $\left|x_{0}\right|$ is arbitrarily large, $\left|x_{0}\right|>M$ for any $M$. But since $w_{\lambda}\left(x_{0}\right)<0$, further $x_{0} \in \Sigma_{\lambda}$, we have $u\left(\left[x_{0}\right]_{\lambda}\right)<u\left(x_{0}\right)$. Thus $\psi\left(x_{0}\right) \leqq u\left(x_{0}\right)$, and thus $e^{\psi\left(x_{0}\right)} \leqq e^{u\left(x_{0}\right)}$. Therefore, as $\bar{V}$ satisfies (HP1)-(HP5), by Corollary $1, e^{u\left(x_{0}\right)} \leqq C\left|x_{0}\right|^{-3-\varepsilon}$. Thus for $\left|x_{0}\right|$ large, as $\bar{V} \in L^{\infty}$,

$$
\frac{\Delta g}{g}+\bar{V}\left(x_{0}\right) e^{\psi\left(x_{0}\right)} \leqq \frac{\Delta g}{g}+C\left|\bar{V}\left(x_{0}\right)\right|\left|x_{0}\right|^{-3-\varepsilon}<0 .
$$

At a minimum point, $\nabla w_{\lambda}\left(x_{0}\right)=0$. Whence from (2.14) and (2.16) we get that $\Delta w_{\lambda}\left(x_{0}\right)<0$ as $w_{\lambda}\left(x_{0}\right)<0$ by assumption. But at a minimum point $x_{0}$, $\Delta w_{\lambda}\left(x_{0}\right)>0$. Thus we have produced a contradiction. Q.E.D.

Remark. If it is known that $V<0$ for $r$ large, or $V \downarrow 0$ sufficiently fast, then 1.h.s. $(2.16)<0$ for $\left|x_{0}\right|$ large. Corollary 1 is superfluous in these cases, and (HP4) can be dropped.

\section{Proof of Theorem P1}

We are now ready to prove Theorem $\mathrm{P} 1$.

Proof. First note that $w_{\lambda}(x) \geqq 0$ for $\lambda<-M, M$ large. This is because by Lemma $1, w_{\lambda}(x) \rightarrow 0$ uniformly as $|x| \rightarrow \infty$. Indeed, if there were a $\tilde{x}$, with $|\tilde{x}|$ large, for which $w_{\lambda}(\tilde{x})<0$, then a strictly negative global minimum of $w_{\lambda}(x)$ for large $|x|$ would result, which is ruled out by Lemma 3 . So $w_{\lambda}(x) \geqq 0$ for $\lambda<-M$, $M$ large.

We now slide the line $T_{\lambda}$ (recall that $x_{1}=\lambda$ on $T_{\lambda}$ ) to the right until we reach a critical value $\lambda_{0}$. This $\lambda_{0}$ is the largest value of $\lambda$ for which $w_{\lambda}(x) \geqq 0, x \in \Sigma_{\lambda}$. 
Claim A: $v_{\lambda}(x)=u_{\lambda}(x)-u(x)>0$ for $x \in \Sigma_{\lambda}, \lambda<\lambda_{0}$, and $\partial_{x_{1}} u>0$ for $x_{1}<\lambda_{0}$.

Claim $B: \lambda_{0}=-4$.

Proof of Claim A. Recall the maximum principle (MP) and the Hopf maximum principle (HMP) [GT]:

Let $\Delta v+b_{i}(x) \partial_{x_{i}} v+c(x) v \leqq 0$ in $\Omega \subset \mathbb{R}^{n}$ and $v \geqq 0$. (Here we use summation convention.)

MP: If $v(\hat{x})=0$ for at least one $\hat{x} \in \operatorname{int}(\Omega)$, then $v \equiv 0$ in all of $\Omega$.

HMP: If $v \not \equiv 0$ in $\Omega, v \geqq 0$, and $\partial \Omega$ is smooth with $\left.v\right|_{\partial \Omega} \equiv 0$, then $\partial v / \partial v<0$, where $\partial v / \partial v$ is the exterior normal derivative on $\partial \Omega$. (Notice that no sign condition is being imposed on $c(x)$ as the minimum of $v$ is 0 .)

We begin by establishing Claim (A). Suppose, for $\lambda<\lambda_{0}$, that $v_{\lambda}(x)=0$ at some point $x \in \Sigma_{\lambda}$. Since $w_{\lambda}(x) \geqq 0$, for $x \in \Sigma_{\lambda}$ and $g(x)>0$, we see that $v_{\lambda}(x) \geqq 0$ in $\Sigma_{\lambda}$. Thus if $v_{\lambda}(x)=0$ the minimum of $v_{\lambda}(x)$ is achieved in $\Sigma_{\lambda}$. Since (2.13) holds, and $v_{\lambda}(x) \geqq 0$, we can apply the maximum principle and deduce $v_{\lambda}(x) \equiv 0$ in $\Sigma_{\lambda}$. This means for $\lambda=\lambda_{0}-\delta$, some $\delta>0$, that $u\left(\lambda_{0}-2 \delta, y\right)=u\left(\lambda_{0}, y\right)$. But $v_{\lambda}(x) \geqq 0$, thus $u\left(x_{\lambda}\right) \geqq u(x)$, which implies $\partial u / \partial x_{1} \geqq 0$ for $x_{1} \leqq \lambda_{0}$. This fact together with the fact $u\left(\lambda_{0}-2 \delta, y\right)=u\left(\lambda_{0}, y\right)$ yields $\partial u / \partial x_{1}=0$ for $\lambda_{0}-2 \delta \leqq x_{1} \leqq \lambda_{0}$. In particular, $\partial u / \partial x_{1}=0$ when $x_{1}=\lambda_{0}-2 \delta$. By the Hopf maximum principle and the maximum principle we have $v_{\lambda} \equiv 0$ iff $\partial v_{\lambda} / \partial x_{1}=0$ on $\left\{x_{1}=\lambda\right\}$. Now $\partial v_{\lambda} / \partial x_{1}=-2 \partial u / \partial x_{1}$ for $x_{1}=\lambda$. But since $\partial u / \partial x_{1}=0$ when $x_{1}=\lambda_{0}-2 \delta$, we see $\partial v_{\lambda_{0}-2 \delta} / \partial x_{1}=0$ for $x_{1}=\lambda_{0}-2 \delta$ or, which is the same, on $T_{\lambda_{0}-2 \delta}$. Thus the Hopf maximum principle says $v_{\lambda_{0}-2 \delta} \equiv 0$. We may repeat this procedure indefinitely and thus deduce that $u$ is independent of $x_{1}$. This is a contradiction, and so the first assertion of claim (A) is proved.

As for the second assertion of (A), note that since $v_{\lambda}>0$ in $\Sigma_{\lambda}$ for $\lambda<\lambda_{0}$, and $v_{\lambda}=0$ on $T_{\lambda}$, we get the Hopf maximum principle $\partial v_{\lambda} / \partial x_{1}<0$ on $T_{\lambda}$. Since for $x_{1}=\lambda$ we have $\partial u / \partial x_{1}=-(1 / 2) \partial v_{\lambda} / \partial x_{1}$, we also have $\partial u / \partial x_{1}>0$ for $x_{1}=\lambda$, with $\lambda<\lambda_{0}$. So claim (A) is proved.

We now prove (B). From the second assertion in claim (A) we see $u$ is strictly increasing for $x_{1}<\lambda_{0}$. By our earlier choice of coordinate axis the maximum of $u$ is at $(-4,0)$. Thus $\lambda_{0} \leqq-4$. We now consider the case $\lambda_{0}<-4$. There are two possibilities. Either $w_{\lambda_{0}} \equiv 0$ or $w_{\lambda_{0}} \not \equiv 0$. We will first rule out the case $\lambda_{0}<-4$, $w_{\lambda_{0}} \not \equiv 0$.

By (2.14) and the maximum principle, as $w_{\lambda_{0}} \equiv 0$ on $T_{\lambda_{0}}$, we get $w_{\lambda_{0}}>0$ for $x_{1}<\lambda_{0}$. Hence, by the Hopf maximum principle, $\partial w_{\lambda_{0}} / \partial x_{1}<0$ when $x_{1}=\lambda_{0}$. On the other hand, by definition of $\lambda_{0}$, there exists a sequence of numbers $\lambda_{k}$, decreasing to $\lambda_{0}$, such that $w_{\lambda_{k}}<0$ and $\lambda_{0}<\lambda_{k}<-3$. Notice $w_{\lambda_{k}}$ is well defined for $\lambda_{k}<-3$. Let $x_{k}$ be a minimum point for $w_{\lambda_{k}}$. Then $w_{\lambda_{k}}\left(x_{k}\right)<0$ and $\nabla w_{\lambda_{k}}\left(x_{k}\right)=0$. As Lemma 3 implies $\left|x_{k}\right|<R_{0}$, there exists a subsequence $x_{k_{j}} \rightarrow x_{0}$ such that $\nabla w_{\lambda_{0}}\left(x_{0}\right)=0$ and $w_{\lambda_{0}}\left(x_{0}\right) \leqq 0$ for $x_{0}=(A, B), A \leqq \lambda_{0}$. This is a contradiction. Thus our claim is proved in this case.

We will next rule out the case $\lambda_{0}<-4$ and $w_{\lambda_{0}} \equiv v_{\lambda_{0}} \equiv 0$. This means $u\left(x_{\lambda_{0}}\right)=u(x)$, for $x \in \Sigma_{\lambda_{0}}$. But $u$ attains its maximum at $(-4,0)$ and by part (A) of our claim, $\partial u / \partial x_{1}>0$ for $x_{1}<\lambda_{0}$. Since $\lambda_{0}<-4$, it follows $\partial u / \partial x_{1}=0$ at $\left(4+2 \lambda_{0}, 0\right)$, which again is a contradiction.

Thus $w_{\lambda_{0}}(x) \geqq 0$ for $\lambda_{0}=-4$, whence $v_{\lambda_{0}} \geqq 0$ or, which is the same, $u\left(x_{\lambda_{0}}\right) \geqq u(x)$ for $\bar{\lambda}_{0}=-4$, where $u$ has a maximum at $(-4,0)$. We may now repeat this argument by sliding the "plane" $T_{\lambda}$ in from $x_{1}=\infty$ to get $u\left(x_{\lambda_{0}}\right) \leqq u(x)$ for 
$\lambda_{0}=-4$. Putting the two inequalities together we see that for $\lambda_{0}=-4$ we have $u\left(x_{\lambda_{0}}\right)=u(x)$. This now implies that $u$ is radially symmetric about $(-4,0)$.

Next we notice that, if $\bar{V}$ were given by $\bar{V}(x)=\mu^{-2} V(|x| / \mu)$, then the radial symmetry of $u$ and the fact that $u$ satisfies (2.11) imply that $V$ is radially symmetric about $(-4,0)$. This can only happen if $V$ is a constant (since $V$ is radially symmetric around the origin by assumption). Thus $\bar{V}$ has to be a constant or of the form $V\left(x_{1}+4, x_{2}\right)$, for non-constant $V$. In both cases, through translating axes back, the solution of the original equation for $u$, i.e. which satisfies $\left(1.3^{p}\right)$, is radially symmetric around the origin. Moreover, from the arguments involving the Hopf maximum principle we see that any solution is also radial decreasing. This completes the proof of Theorem P1. Q.E.D.

\section{Proofs of Theorems P2, S1, S2}

In this section we show first that by stereographic projection our Theorem $\mathrm{P} 1$ about $\left(1.3^{p}\right)$ in $\mathbb{R}^{2}$ implies Theorem $S 1$ about $\left(1.3^{s}\right)$ on $S^{2}$. We then prove in the same fashion Theorem S2, and finally Theorem P2.

Proof of Theorem $S 1$. Consider $S^{2}$ to be embedded in $\mathbb{R}^{3}$ and centered at the origin. Then $S^{2}=\left\{y^{(1)}, y^{(2)}, y^{(3)} \mid \sum_{i} y^{(i)^{2}}=1\right\}$. We project $S^{2}$ stereographically onto $\mathbb{R}^{2}=\left\{y^{(1)}=x^{(1)}, y^{(2)}=x^{(2)}, y^{(3)} \mid y^{(3)}=0\right\}$. Points in $\mathbb{R}^{2}$ will be denoted by $x$. We assume without loss of generality that the symmetry axis $\mathbf{n}$ of $V(y), y \in S^{2}$, is the north pole $(0,0,1)$. We set $V(y)=\hat{V}(\langle\mathbf{n}, y\rangle)$. We have $\langle\mathbf{n}, y\rangle=1-|J|$, with

$$
|J|(r)=\frac{2}{1+r^{2}}
$$

and $r=|x|, x \in \mathbb{R}^{2}$. Note that $|J|^{2}$, the Jacobian of the stereographic projection $S^{2} \rightarrow \mathbb{R}^{2}$, is radial decreasing. Thus $\left(1.3^{s}\right)$ becomes

$$
-\Delta u=|J|^{2}\left[\hat{V}(1-|J|) e^{u}-c\right] .
$$

In (4.2), $\Delta$ is the Laplace operator on $\mathbb{R}^{2}$, and in $u$ the $y \in S^{2}$ are to be expressed as functions of the Euclidean variables.

Next we introduce

$$
v(x)=u[y(x)]+c \ln |J| .
$$

A straightforward integration yields

$$
-\Delta v=V_{p}(r) e^{v}
$$

with

$$
V_{p}(r)=|J|^{2-c} \hat{V}(1-|J|) .
$$

Next, notice that $1-|J|$ is increasing as a function of $r$ and that, by (HS2), $t \mapsto \hat{V}(t)$ is nonincreasing, so $\hat{V}(1-|J|)$ is nonincreasing as a function of $r$. Obviously, for $c \leqq 2, V_{p}$ is nonincreasing as a function of $r$ if $\hat{V} \geqq 0$. Equation $\left(1.3^{s} \mathrm{~b}\right)$ together with (HS3) implies $c \leqq 2$, thus $V_{p}$ is nonincreasing as a function of $r$ by (HS1) and (HS3). By substitution of variables, since (HS4) holds by assumption, then (HP4) holds for $\int V_{p} e^{v} d^{2} x$. Radial symmetry of $V_{p}$ holds by construction, and the $L^{1}$ bound (HP3) for $V_{p} e^{v}$ holds by substitution of variables as the requirement 
(HS3) holds. Similarly (HP5) holds for $v^{+}$as (HS5) holds for $u$, by assumption. Therefore Theorem S1 holds as a consequence of Theorem P1. Q.E.D.

Proof of Theorem S2: In the first step we define $\mathbf{n}$ to be the north pole and then proceed as in the proof of Theorem S1. This time, however, the stereographic projection results in an equation $\left(1.3^{p}\right)$ with weight

$$
V_{p}(x)=U[y(x)],
$$

and the decrease and boundedness of $V_{p}$, demanded by (HP1) and (HP2), follow from (HS1*) and (HS2*). (HP3) and (HP4) are conformal to (HS3*) and (HS4*). Finally, (HP5) is satisfied by substitution of variables. This proves Theorem S2 for n being the north pole.

In the second step, we notice that nobody knows which direction is "north" on $S^{2}$, which proves Theorem S2. Q.E.D.

Proof of Theorem P2. Notice that a Kelvin transform $x \rightarrow x /|x|^{2}$ in $\mathbb{R}^{2}$ is equivalent to $\mathbf{n} \rightarrow-\mathbf{n}$ on $S^{2}$. We pick the situation covered by Theorem $\mathrm{S} 2$ and choose $\mathbf{n}$ to be the south pole. Then we proceed as before. The resulting stereographically projected situation is the one covered by Theorem P2. Q.E.D.

We remark that automatically $m \leqq 4$ in $\left(\mathrm{HP} 1^{*}\right)$, and $c \leqq 2$ in $\left(\mathrm{HS} 1^{*}\right)$. We like to thank J.J. Aly for suggesting to us to combine our Lemma 1 and Pohozaev's identity to extend [CLMP] (7.6). Indeed, one then concludes a priori that $m \leqq 4$ for any solution of $\left(1.3^{p}\right)$ with finite generating mass provided (HP1) and (HP2) are met. Therefore, Theorem $\mathrm{P} 2$ does not cover $V$ which are asymptotically increasing according to a power law.

\section{A Sufficiency Condition on $V$ Which Implies (HP4)}

We are now proving a sufficient condition for $V$ so that all solutions of $\left(1.3^{p}\right)$ satisfy (HP4).

Proposition 1. Suppose there exists constants $c_{1}, c_{2}=\operatorname{ess} \sup V$, with $c_{1} / c_{2}>3 / 4$, such that $0<c_{1} \leqq V(x) \leqq c_{2}<\infty$ for $x \in \mathbb{R}^{2}$. Then any solution of $\left(1.3^{p}\right)$ satisfies

$$
\int_{\mathbb{R}^{2}} V(x) e^{u(x)} d^{2} x>6 \pi .
$$

Proof. First we introduce $U(x)$ via $V(x)=\|V\|_{\infty} U(x)$ and $w(x)=u(x)+\ln \|V\|_{\infty}$. By the demanded bounds on $V$, we have $3 / 4<U(x) \leqq 1$. Moreover, since $u$ satisfies $\left(1.3^{p}\right)$ by assumption, $w$ satisfies

in all $\mathbb{R}^{2}$.

$$
-\Delta w=U(x) e^{w}
$$

We now proceed by a modification of an idea of Ding, see [CheL]. For $w$ a solution of (5.1), let $w^{*}$ denote the non-increasing radial rearrangement of $w$. Let $\Lambda_{c}=\{x \mid w(x) \geqq c\}$, and let $B_{R_{c}}(0)$ denote the ball with radius $R_{c}$ centered at the origin, with $R_{c}$ chosen such that $\left|\Lambda_{c}\right|=\left|B_{R_{c}}\right|$. By the isoperimetric inequality,

$$
-\int_{\partial B_{R_{c}}(0)} \partial_{|x|} w^{*} d s \leqq-\int_{\partial \Lambda_{c}} \mathbf{n} \cdot \nabla w d s=-\int_{\boldsymbol{\Lambda}_{c}} \Delta w d^{2} x
$$


By virtue of the fact that $w$ solves (5.1) and the bounds on $U$,

$$
-\int_{\Lambda_{c}} \Delta w d^{2} x=\int_{\Lambda_{c}} U e^{w} d^{2} x \leqq \int_{\Lambda_{c}} e^{w} d^{2} x=\int_{B_{R_{c}}(0)} e^{w^{*}} d^{2} x
$$

Let now

$$
f(r)=\int_{B_{r}(0)} e^{w^{*}} d^{2} x
$$

Then $f^{\prime}(r)=2 \pi r e^{w^{*}(r)}$ and $f^{\prime \prime}(r)=2 \pi\left[r w^{*}(r)^{\prime}+1\right] e^{w^{*}(r)}$. With the aid of (5.2), (5.3) and (5.4) we see $-2 \pi r w^{*}(r)^{\prime} \leqq f(r)$. Thus $2 \pi r f^{\prime \prime}(r) \geqq 2 \pi f^{\prime}(r)-f(r) f^{\prime}(r)$. Since $f^{\prime}(0)=0$, integration by parts leads us to $4 \pi r f^{\prime}(r) \geqq 8 \pi f(r)-f^{2}(r)$. Next, with $\phi(r)=1 / f(r)$, we get from the last inequality $\left[r^{2} \phi(r)\right]^{\prime} \leqq r / 4 \pi$ which upon integration immediately yields

$$
\int_{\mathbb{R}^{2}} e^{w} d^{2} x \geqq 8 \pi
$$

Thus, since $U>3 / 4$ by the assumed bounds on $V$, we get

$$
\int_{\mathbb{R}^{2}} U(x) e^{w} d^{2} x>(3 / 4) 8 \pi=6 \pi
$$

However, by construction

$$
\int_{\mathbb{R}^{2}} V(x) e^{u} d^{2} x=\int_{\mathbb{R}^{2}} U(x) e^{w} d^{2} x,
$$

and the proposition is proved. Q.E.D.

Remark. Note that in Proposition 1 it is not assumed that $V$ is radial monotonic decreasing. Note also that by assumption $V$ is bounded away from 0 , so that in this case we can drop both (HP4) and (HP5); see the corresponding remark in the introduction.

\section{An Example of Broken Symmetry}

In Sect. 4 we saw that for $c>2$ the stereographic projection $S^{2} \rightarrow \mathbb{R}^{2}$ maps a $\hat{V}$ from problem $\left(1.3^{s}\right)$ to a problem $\left(1.3^{p}\right)$ with a $V(x)$ that contains an additional factor $\left(1+r^{2}\right)^{c-2}$; see (4.5). For strictly positive $\hat{V}$ which are also bounded away from zero, the so generated $V(x)$ increases asymptotically like a power law for $r$ large. This violates both (HP1) and (HP2). Since this case is not covered by our Theorems P1, $\mathrm{P} 2$, it is of interest to clarify whether this implies a breaking of the radial symmetry.

In this section we consider the slightly simpler but related problem of radial symmetric $V(x)$ which increase according to a pure power law. The essential feature of a radial $V$ which violates (HP1) and (HP2) is thus kept. Explicit counterexamples to radial symmetry which satisfy all hypothesis but (HP1) and (HP2) will now be constructed.

Proposition 2. For $k \in \mathbb{N}$, let $V(x)$ be given by $V_{k}(x)=8 k^{2} r^{2(k-1)}$, where $r=|x|$, $x \in \mathbb{R}^{2}$. Let $\varphi$ be the polar angle in $\mathbb{R}^{2}, \varphi_{0}$ some reference angle, and let $r_{0}$ be a reference scale. Then for each $\zeta \in \mathbb{R}, r_{0} \in \mathbb{R}^{+}, \varphi \in[0,2 \pi)$, the function $W_{\zeta}^{(k)}\left(r, \varphi ; r_{0}, \varphi_{0}\right)$ given by

$$
\begin{aligned}
\exp \left[W_{\zeta}^{(k)}\left(r, \varphi ; r_{0}, \varphi_{0}\right)\right]= \\
\quad r_{0}^{-2 k}\left\{1+2\left(r / r_{0}\right)^{k} \cos \left(k\left[\varphi-\varphi_{0}\right]\right) \tanh \zeta+\left(r / r_{0}\right)^{2 k}\right\}^{-2} \operatorname{sech}^{2} \zeta
\end{aligned}
$$


is a $C^{\infty}\left(\mathbb{R}^{2}\right)$ solution of $\left(1.3^{p}\right)$ with $V=V_{k}$. In particular, (6.1) satisfies (HP3) and (HP4). For $\zeta=0$, any $k \in \mathbb{N}$, the solution (6.1) is radially symmetric around the origin. For $k=1$, and $\zeta \neq 0,(6.1)$ is radially symmetric about some $x_{0} \in \mathbb{R}^{2}$ with $\left|x_{0}\right|=\tanh |\zeta|$. For $k>1$ and $\zeta \neq 0, W_{\zeta}^{(k)}$ is not rotationally symmetric about any point in $\mathbb{R}^{2}$. We have

$$
\int_{\mathbb{R}^{2}} 8 k^{2} r^{2(k-1)} \exp \left[W_{\zeta}^{(k)}(x)\right] d^{2} x=8 \pi k
$$

independent of $\zeta, r_{0}$ and $\varphi_{0}$. Also, $W_{\zeta}^{(k)^{+}}(x)$ is compactly supported in a simply connected bounded domain; hence (HP5) holds, too.

Proof. It is straightforward to insert (6.1) into $\left(1.3^{p}\right)$, using the definition of $V_{k}$ and to verify that it is a solution. It is however more instructive to see how it is constructed ab initio.

We construct $(6.1)$ from (1.4), the periodic solution of $\left(1.3^{p}\right)$ for constant $V_{(14)}=1$, i.e. Liouville's equation. With the transformations $\xi=\ln r$ we have

$$
\Delta=r^{-1} \partial_{r}\left(r \partial_{r}\right)+r^{-2} \partial_{\varphi \varphi}=r^{-2}\left(\partial_{\xi \xi}+\partial_{\varphi \varphi}\right) \text {. }
$$

If we interpret $\xi$ and $\varphi$ as cartesian coordinates of $\mathbb{R}^{2}$, then $U_{\zeta, \kappa}\left(x ; x_{0}\right)$ from (1.4) with $\langle\mathbf{v}, x\rangle=\xi,\left\langle\mathbf{v}^{\prime}, x\right\rangle=\varphi$, satisfies Liouville's equation in these coordinates, and so, using (6.3) and transforming back to $r, \varphi$ coordinates, and further absorbing a numerical factor into the emerging $V$, we arrive at a formal solution of $\left(1.3^{p}\right)$ with formal potential $\tilde{V}_{\kappa}=8 \kappa^{2} r^{-2}$. We say formal as we have yet to check the singular behavior of this solution. First it is clear that, in order to get a single valued function, $\kappa$ has to be restricted to positive integers $k \in \mathbb{N}$, since $\varphi$ is a polar angle in the left-hand side of (6.3). Second, it is easy to check that this solution generates a Dirac singularity at the origin, with mass $-4 \pi k$, therefore we have so far only arrived at a solution of $\left(1.3^{p}\right)$ in $\mathbb{R}^{2} \backslash\{0\}$. To get a solution for all $\mathbb{R}^{2}$ we add the corresponding Green's function $-2 k \ln r$ to our formal solution, lump the compensating $r^{2 k}$ together with $\tilde{V}_{k}$ to a new $V_{k}$ and arrive at (6.1).

The rotational symmetry in the case that $\zeta=0$ is obvious. It is left as an exercise to verify that for $k=1$ our (6.1) is just a rotationally symmetric solution (1.2c) of (1.2a), with the identification $\lambda^{-2}=\cosh ^{2} \zeta,\left|x_{0}\right|=\tanh |\zeta|$, and with $\left\langle x, x_{0}\right\rangle=$ $r\left|x_{0}\right| \cos \left(\varphi-\varphi_{0}\right)$. (This means that the variable $r$ here is not the radial variable measured from the center of symmetry of $W_{\zeta}^{(k)}$ for $k=1$.) For $k>1$, we have solutions which are nontrivially periodic in $\varphi$ provided $\zeta \neq 0$.

A standard calculation shows that

$$
r^{2(k-1)} \exp \left(W_{\zeta}^{(k)}\right) \sim r^{2(k-1)}
$$

for $r \rightarrow 0$, and

$$
r^{2(k-1)} \exp \left(W_{\zeta}^{(k)}\right) \sim r^{-2(k+1)}
$$

as $r \rightarrow \infty$, and (6.1) is $C^{\infty}$ for $r \in[0, \infty)$. This proves that (HP3) is satisfied. Obviously $W_{\zeta}^{(k)} \rightarrow-2 \ln \left(r_{0}^{k} \cosh \zeta\right)$ for $r \rightarrow 0^{+}$and $W_{\zeta}^{(k)} \rightarrow-\infty$ for $r \rightarrow \infty$, so $W_{\zeta}^{(k)^{+}}$is compactly supported on a bounded simply connected domain. Hence (HP5) holds true.

Knowing this, we can calculate the total mass generated by the solutions by using that $W_{\zeta}^{(k)}$ solves $\left(1.3^{p}\right)$ with $V=8 k^{2} r^{2(k-1)}$, and partial integration. We get

$$
\int_{\mathbb{R}^{2}} 8 k^{2} r^{2(k-1)} \exp \left(W_{\zeta}^{(k)}\right) d^{2} x=-\left.\lim _{r \rightarrow \infty} r \int_{\partial B_{r}(0)} \partial_{t} W_{\zeta}^{(k)}(t, \varphi)\right|_{r} d \varphi=8 \pi k
$$


Thus (HP3) holds too, as well as (HP4). For $k=1$, i.e., for the rotationally symmetric solutions, we recover the total mass of (1.2c). Q.E.D.

To the best of our knowledge, this is the first counterexample to radial solutions of a radially symmetric equation in $\mathbb{R}^{2}$ of the type $\left(1.3^{p}\right)$ which has finite mass. In the next section, where we apply our results to some problems in physics and geometry, we will see that the symmetry breaking (6.1) indicates the possibility of a new phase transition in vortex gases.

\section{Applications}

We now discuss three applications of our results to physics and geometry.

7a. Planar Onsager Vortex Theory. The thermodynamically unstable vortex Hamiltonian

$$
H=-(4 \pi)^{-1} \sum_{i \in \mathscr{I}} \sum_{j \in \mathscr{I} \backslash\{i\}} \ln \left|x_{i}-x_{j}\right|
$$

with $x_{i} \in \mathbb{R}^{2}, i \in \mathscr{I}=\{1,2, \ldots, N\}$ admits as further constant of motion

$$
I=\sum_{i}\left|x_{i}\right|^{2}
$$

which effectively confines the vortices. (In $\mathbb{R}^{2}$, the center of vorticity is a trivial further constant of motion, which we imagine to be at the origin by a simple translation.) One is interested in constructing directly the corresponding "bi"microcanonical ensemble (MCE) in a thermodynamic mean-field limit, which is expected to have importance in two-dimensional turbulence; see [Ons] for the original idea and [MMSMO] for recent simulations on the 2-torus $T^{2}$. The direct construction of the MCE for (7a.1) is an open problem in general; see below. So far one relies on the weaker bi-canonical ensemble (CE) for which better control is available, which allows one to construct rigorously the thermodynamic mean-field limit as in [K, CLMP] for the joint $N$ vortex distribution $\mu^{(N)}\left(d^{2 N} x\right)=$ $Z^{-1} \exp \{-H / T-\gamma \mathrm{I}\} d^{2 N} x$, with temperature scaled like $T(N)=\beta^{-1} N, \beta$ fixed, and $\gamma>0$ the conjugate variable to $I$. As $N \rightarrow \infty$, for all $\beta>-8 \pi$ and all $\gamma>0, L^{p}$ weak limit points of the marginal probability measures $\mu_{n}^{(N)}$ exist for $p \in[1, \infty)$. Any weak limit point $\mu_{n}$ is a convex superposition of marginals of infinite product measures $\rho^{\otimes \mathbb{N}}$ which satisfy

$$
\begin{aligned}
\rho(x) & =\frac{\exp \left(-\beta \phi-\gamma r^{2}\right)}{\int_{\mathbb{R}^{2}} \exp \left(-\beta \phi-\gamma r^{2}\right) d^{2} x}, \\
-\Delta \phi & =\rho .
\end{aligned}
$$

In the bi-canonical ensemble, only solutions of $(7 \mathrm{a} .3 \mathrm{a} / \mathrm{b})$ which are global minimizers of ( $\beta$ times) the free energy per pair of particles,

$$
\beta F(\rho)=\int_{\mathbb{R}^{2}} \rho(x)\left[\frac{-\beta}{4 \pi}(\ln |x| * \rho)(x)+\ln \rho(x)+\gamma|x|^{2}\right] d^{2} x
$$

are admitted in the convex superposition. Here $*$ denotes convolution. In case (7a.4) has a unique minimizer the superposition measure is a singleton, implying in fact weak $L^{p}$ convergence of the marginals as $N \rightarrow \infty$. The limiting measures are in 
$L^{\infty}$. Note that our statement implies that a finite global minimum on $L^{1} \cap L^{\infty}\left(\mathbb{R}^{2}\right)$ of (7a.4) does exist for $\beta>-8 \pi ; \gamma>0$, see [K, CLMP]. For the canonical ensemble it is enough to determine and evaluate all minimizers of (7a.4) for $\beta>-8 \pi$. For the microcanonical ensemble this is not a-priori clear, as $\beta$ is a secondary quantity in the MCE and may well be below $-8 \pi$, for instance, and the corresponding solution of $(7 \mathrm{a} .3 \mathrm{a} / \mathrm{b})$ may not be a global minimizer of (7a.4) then. We remark that in a finite disk this happens, see [SO'N] for numerical Monte Carlo results and for perturbation theoretic results.

Concerning symmetry of canonical minimizers, a number of results follow from the investigation of [CLMP]. For $\beta>0, \rho \mapsto \beta F$ is neither a sum of convex functionals (as $-\ln |x-y|$ is not a positive kernel) nor monotone under symmetric decreasing rearrangement. For $\beta>0$ (7a.4) is however almost convex, suggesting a unique, hence radial, solution of $(7 \mathrm{a} .3 \mathrm{a} / \mathrm{b})$. For $-8 \pi<\beta<0,(7 \mathrm{a} .4)$ is decreasing under symmetric decreasing rearrangements, which implies radial symmetry of the minimizers in that range of $\beta$ values. These arguments are essentially due to [CLMP], proof of Theorem 6.1, with some slight modifications; cf. [CLMP] Sect. 6. Caglioti et al. also showed by a standard ODE argument that radially symmetric solutions exist, moreover that they are unique for each $\beta>-8 \pi, \gamma>0$, and further that all radial solutions satisfy $\beta>-8 \pi$. It is then possible to conclude the same symmetry and uniqueness results for the microcanonical entropy maximizers by a convexity argument, as in Caglioti's thesis, cf. [ES].

The next objects to inquire into after the canonical and microcanonical extremizers are possible metastable solutions. From the work of [CLMP] it is not clear a-priori whether all solutions of $(7 \mathrm{a} .3 \mathrm{a} / \mathrm{b})$ are radial also for all $\beta<0$, and especially whether nonradial solutions exist for $\beta<-8 \pi$. Nonradial solutions would come in a family, as the orientation must be degenerate. This would remind us of a symmetry breaking phase transition, though at most for metastable states. Nevertheless it is an attractive possibility to inquire into. Restricting (7a.3a/b) to a finite disk with 0-Dirichlet b.c., such a phase transition was found both numerically and with perturbation techniques in [SO'N]. Notice however that 0-Dirichlet b.c. for $\phi$ mimick an adjustable environment (image "charges") so that a catalyzer effect for symmetry breaking prevails. On the basis of numerical computations Williamson [Will] conjectured that only radial solutions of $(7.3 \mathrm{a} / \mathrm{b})$ exist in $\mathbb{R}^{2}$.

Our Theorem P1 confirms Williamson's conjecture for all $\beta<-6 \pi$. This can be expressed as

Corollary P1. All nondistributional solutions of $(7 \mathrm{a} .3 \mathrm{a} / \mathrm{b})$ in the regime $\beta<-6 \pi$ are necessarily radial symmetric and decreasing.

We can go further and confirm Williamson's conjecture for all $\beta \leqq 0$. We notice that here we have an example where we gave away some information about our $V$, which is decreasing rapidly to zero. By inspection of the proofs of Theorem P1 and Lemma 3, we see that we can drop (HP4) [see the remark after Lemma 3] and thus have proved

Theorem VI. All nondistributional solutions of $(7 \mathrm{a} .3 \mathrm{a} / \mathrm{b})$ in the regime $\beta \leqq 0$ are necessarily radial symmetric and decreasing.

Notice that this goes further than the radial symmetry of only all minimizers of (7a.4) for $\beta \in(-8 \pi, 0)$, which exist by [K] and [CLMP], and which are radial by 
symmetric decreasing rearrangement; see above. Since by Eq. (7.6) of [CLMP] all radial solutions satisfy $\beta>-8 \pi$, we can conclude also from Corollary P1 that $(7 \mathrm{a} .3 \mathrm{a} / \mathrm{b})$ has no nondistributional solutions for $\beta \leqq-8 \pi$. For further rigorous results on the planar vortex problem (7a.3) in $\mathbb{R}^{2}$ we refer to [CLMP and $\mathrm{CK}]$.

We see that to have the possibility of symmetry breaking in $\mathbb{R}^{2}$ we have to admit distributional solutions of $(7 \mathrm{a} .3 \mathrm{a} / \mathrm{b})$ or relax some other hypothesis.

An interesting situation arises if we extend (7a.1) and assume that the vortices are subjected to a fixed external stream field $\Phi$ which is radial in $\mathbb{R}^{2}$. We remark that this fixed field cannot act as a catalyzer of symmetry breaking in the sense in which 0-Dirichlet b.c. for $\phi$ do. We expect it difficult to find a symmetry breaking if $\Phi$ is bounded. The situation changes if we take as the source for $\Phi$ a point vortex of opposite sign and strength $N M>0$ at the origin. In the mean-field limit we have to consider

If

$$
\begin{gathered}
\rho(x)=\frac{r^{-\beta M / 2 \pi} \exp \left(-\beta \phi-\gamma r^{2}\right)}{\int_{\mathbb{R}^{2}} r^{-\beta M / 2 \pi} \exp \left(-\beta \phi-\gamma r^{2}\right) d^{2} x}, \\
-\Delta \phi=\rho .
\end{gathered}
$$

$$
M=\frac{k-1}{2 k}
$$

with $k \in \mathbb{N}, k>1$, then for $\gamma=0$ and $\beta=-8 \pi k$, we obtain solutions with broken symmetry from (6.1). We leave it as an exercise to rewrite (6.1) into a solution of (7a.5). Questions of stability will be discussed in [CK].

7.b Spherical Onsager Vortex Theory. As a second example, which bridges the "gap" between statistical mechanics of classical vortices and the random surface problem studied by Onofri [On, OV], consider the Hamiltonian

$$
H=-(8 \pi)^{-1} \sum_{i \in \mathscr{I}} \sum_{j \in \mathscr{I} \backslash\{i\}} \ln \left(1-\left\langle y_{i}, y_{j}\right\rangle\right)
$$

with $y_{i} \in S^{2}, i \in \mathscr{I}=\{1, \ldots, N\}$. Here the angular brackets denote scalar product in $\mathbb{R}^{3}$, in which we imagine $S^{2}$ to be embedded. The Hamiltonian (7b.1) gives rise to point vortex motion on the 2-sphere [PD] when the canonical variables are given by $\left\langle\mathbf{p}, y_{i}\right\rangle$ and $\varphi_{i}$, with $\mathbf{p} \in S^{2}$ fixed and $\varphi_{i}$ the polar angle around $\mathbf{p}$ as measured from some fixed meridian through $\mathbf{p}$. It admits a further constant of motion

$$
D=\sum_{i} y_{i}
$$

which may be termed a vortex dipole moment. As for bounded domains in $\mathbb{R}^{2}$, or all $\mathbb{R}^{2}$, the mean-field limit for the bi-canonical ensemble on $S^{2}$ exists for $\beta>-8 \pi$ in the sense mentioned above. This time

$$
\begin{aligned}
\rho(y) & =\frac{\exp (-\beta \phi-\gamma\langle\mathbf{n}, y\rangle)}{\int_{S^{2}} \exp (-\beta \phi-\gamma\langle\mathbf{n}, y\rangle) d \mu}, \\
-\Delta_{S} \phi & =\rho-1 / 4 \pi,
\end{aligned}
$$


with $\mathbf{n}$ in $S^{2}$, and $d \mu$ the uniform measure on $S^{2}$. Here $\Delta_{S}$ is the Laplace-Beltrami operator on $S^{2}$ with respect to the standard metric. In order to eliminate the zero mode, we add the requirement

$$
\int_{S^{2}} \phi d \mu=0 .
$$

Now $\gamma$ may be positive or negative; however, by a simple rotation we see that without loss of generality we may assume $\gamma \geqq 0$. In the bi-canonical ensemble, only those solutions are admitted which minimize

$$
J(\phi)=\frac{1}{2} \int\left|\nabla_{S} \phi\right|^{2} d \mu+\beta^{-1} \ln \int \exp (-\beta \phi-\gamma\langle\mathbf{n}, y\rangle) d \hat{\mu}+\int \phi d \hat{\mu},
$$

the integrations being over $S^{2}$, with $d \hat{\mu}=(1 / 4 \pi) d \mu$. For the bi-microcanonical ensemble this would generally not be the case. As far as the CE is concerned, for $\beta>0(7 \mathrm{~b} .4)$ is convex, so a unique solution of $(7 \mathrm{~b} .3 \mathrm{a} / \mathrm{b} / \mathrm{c})$ exists, which is thus rotationally symmetric. For $\beta<0$, convexity does not manifestly prevail, leaving the possibility for multiplicity of solutions. For $0>\beta>-8 \pi$, in the canonical ensemble, we use symmetric rearrangements to characterize the minimizers as rotationally symmetric, but that is inappropriate for the microcanonical ensemble, for which also nonminimizing [regarding (7b.4)] solutions of $(7 \mathrm{~b} .3 \mathrm{a} / \mathrm{b} / \mathrm{c})$ are of interest and $\beta>-8 \pi$ is not a-priori required.

Our problem is to find conditions under which all solutions of (7b.3) are rotational symmetric around $\mathbf{n}$. An immediate consequence of Theorem S1 is

Corollary S1. All solutions of $(7 \mathrm{~b} .3 \mathrm{a} / \mathrm{b} / \mathrm{c})$ for $\beta \in[-8 \pi,-6 \pi)$, are necessarily rotational invariant around $\mathbf{n}$.

We notice that again we gave away some information on $V$. We remark that by a more careful inspection of the estimates Lemma 1 and 3, we can drop (HP4) for the stereographically projected problem and arrive at

Theorem V2. All solutions of $(7 \mathrm{~b} .3 \mathrm{a} / \mathrm{b} / \mathrm{c})$ for $\beta \in[-8 \pi, 0]$, are necessarily rotational invariant around $\mathbf{n}$.

This is more than rotational symmetry of only all minimizers of (7b.4) for $\beta \in(-8 \pi, 0)$, which exist by [K] and [CLMP], and are rotation symmetric as explained above. Notice the difference to $\mathbb{R}^{2}$. We remark that the solutions are also monotone decreasing as a function of $\langle\mathbf{n}, y\rangle$, which does not follow from projecting the moving plane results but will be shown in $[\mathrm{CK}]$.

Proof of Theorem V2. Let us for convenience display equations (7b.3a/b/c) stereographically projected on $\mathbb{R}^{2}$. We assume that $\beta<0$ and w.l.o.g. $\gamma \geqq 0$. Then, with $\Delta$ the Laplace operator on $\mathbb{R}^{2}$,

$$
-\Delta \hat{\phi}(x)=\frac{\exp [-\beta \hat{\phi}-\gamma \psi]|J|^{2}}{\int_{\mathbb{R}^{2}} \exp [-\beta \hat{\phi}-\gamma \psi]|J|^{2} d^{2} x}-\frac{|J|^{2}}{4 \pi}
$$

with $\hat{\phi}(x)=\phi(y[x])$ and with $\psi(r)=1-|J|$. Introduce

$$
\tilde{\phi}(x)=-\beta\left[\hat{\phi}(x)-(1 / 4 \pi) \ln \left(1+r^{2}\right)\right]
$$

(recall $\beta<0$, the interesting case). Then

$$
-\Delta \tilde{\phi}(x)=V_{\gamma}(r) e^{\tilde{\phi}(x)}
$$


with

$$
V_{\gamma}(r)=-\beta \frac{|J|^{2+\beta / 4 \pi} \exp [\gamma|J|]}{\int_{\mathbb{R}^{2}}|J|^{2+\beta / 4 \pi} \exp [\tilde{\phi}+\gamma|J|] d^{2} x} .
$$

Now by Lemma 1,

$$
\tilde{\phi}(x) \sim(\beta / 2 \pi) \ln |x|
$$

and therefore

$$
|J|^{2+\beta / 4 \pi} \exp \{\tilde{\phi}(x)\} \sim r^{-4}
$$

for $r$ large. Therefore, 1.h.s. (2.16) $<0$ for $r$ large automatically, hence we can drop (HP4) and still come to the same conclusion as in Lemma 3. The rest of the proof of Theorem V2 is identical to the proof of Theorem P1. Q.E.D.

We recover that our ability to conclude, in Corollary $\mathrm{S} 1$, or Theorem V2, rotational symmetry of solutions of $(7 \mathrm{~b} .3 \mathrm{a} / \mathrm{b} / \mathrm{c})$ only for $\beta \geqq-8 \pi$, comes from the fact that the stereographic projection $S^{2} \rightarrow \mathbb{R}^{2}$, which we employ in the mapping $\left(1.3^{s}\right) \rightarrow\left(1.3^{p}\right)$, generates asymptotically power law increasing $V(x) ; x \in \mathbb{R}^{2}$ for $\beta<-8 \pi$ (we recall $|J| \sim r^{-2}$ ). Thus we expect our example (6.1) of broken symmetry in $\mathbb{R}^{2}$ to be of intrinsic interest also for the vortex, and related, problems on $S^{2}$. Evidently the possibility of a symmetry breaking phase transition in the microcanonical ensemble corresponding to smooth solutions for some $\beta<-8 \pi$ without the help of an external point vortex source is in fact suggested for this model.

This brings us back to the existence problem of the mean-field limit for the MCE. The canonical ensemble requires $\beta>-8 \pi$ as a sharp constraint. To establish the regime $\beta \leqq-8 \pi$ as the limit of finite $N$ problems, the microcanonical ensemble is needed. This more interesting case is in general open. For $\Lambda$ a finite disk in $\mathbb{R}^{2}$, the CE and MCE are equivalent if $\gamma \equiv 0$, in which case Caglioti in his thesis has established the microcanonical mean-field limit by convexity arguments. If one cuts out the logarithmic singularity, more can be said. Here we recommend the recent article by Eyink and Spohn [ES].

7.c Geometry. Next we mention that (7b.3) with $\beta=-8 \pi$ is equivalent to a problem of finding the conformal map that sends the uniform metric on $S^{2}$ into a metric with prescribed Gaussian curvature $\sim \exp (-\gamma\langle\mathbf{n}, y\rangle)$. It is known that this problem has a solution only if $\gamma=0$, see [KW] Theorem 8.8, in which case we are back to Onofri's problem. This is due to the special case of the putative curvature function. We notice that we may admit more general prescribed Gaussian curvature. The main difference between the mean-field limit problem of statistical mechanics and the geometrical problem is that in the latter a Gaussian curvature function is not necessarily everywhere positive. For recent results see [CY].

For a pointwise conformal change of metric on $S^{2}$, we have $\left(1.3^{s}\right)$ with $c=2$. In that case the Gaussian curvature is related to $V(y)$ by $2 K(y)=V(y)=U(y)$, which is therefore monotone. Our Theorem S2 does not a priori demand $V \geqq 0$ everywhere; however, by monotonicity it must be positive in order to be a curvature function on $S^{2}$. And so from Theorem S2 Corollary S2 follows:

Corollary S2. Any conformal map that sends the uniform metric on $S^{2}$ into a metric with prescribed bounded, rotationally symmetric and monotone Gaussian curvature function $K(y) ; y \in S^{2}$, is itself rotational invariant around the same axis. 
Unfortunately this corollary is less illuminating than it pretends to be. By [KW] Eq. (8.11), the only monotone curvature function that admits a solution of $\left(1.3^{s}\right)$ is the constant function. We are indebted to S.Y.A. Chang for recalling to us [KW] (8.11).

Acknowledgement. S. Chanillo expresses his gratitude to K. Jalsur and S.Y.A. Chang for valuable comments. M. Kiessling owes thanks to J.J. Aly, L. Nirenberg, M. Pulvirenti for valuable remarks. The work of S.C. was supported under NSF Grant DMS-9202051; the work of M.K. under NASA Grant NAG3-1414. M. Kiessling would like to thank J. Lebowitz for the kind investigation and warm hospitality at Rutgers, where this work was started, and to J. Percus for generous support during the finalization of this work.

\section{References}

[A] Alexandroff, A.D.: quoted in p. 2 in [BN]

[Al] Aly, J.J.: On the lowest energy state of a collisionless self-gravitating system under phase space volume constraints. M.N.R.A.S. 241, 15-27 (1989)

[Be] Beckner, W. To appear; private communication Feb. 1993

[BM] Brézis, H., Merle, F.: Uniform estimates and blow-up behavior of solutions of $-\Delta u=V(x) e^{u}$ in two dimensions. Commun. PDE 16, 1223-1253 (1991)

[BN] Berestycki, H., Nirenberg, L.: On the method of moving planes and the sliding method. Bol. Soc. Bras. Mat. 22, 1-37 (1991)

[Br] Branson, Th.: The functional determinant. (1992)

[CGS] Caffarelli, L., Gidas, B., Spruck, J.: Asymptotic symmetry and local behavior of semilinear elliptic equations with critical Sobolev growth. Preprint

[CLMP] Caglioti, E., Lions, P.-L., Marchioro, C., Pulvirenti, M.: A special class of stationary flows for two-dimensional Euler equations: A statistical mechanical description. Commun. Math. Phys. 143, 501-525 (1992)

[CaL1] Carlen, E.A., Loss, M.: Extremals of functionals with competing symmetries. J. Funct. Anal. 88, 437-456 (1990)

[CaL2] Carlen, E.A., Loss, M.: Competing symmetries, the logarithmic HLS inequality, and Onofri's inequality on $S^{n}$. Geom. Funct. Anal. 2, 90-104 (1992)

[CK] Chanillo, S., Kiessling, M.: Phase diagrams and other rigorous results in the statistical mechanics of vortex gases. In preparation

[ChaL] Chanillo, S., Li, Y.Y.: Continuity of solutions of uniformly elliptic equations in $\mathbb{R}^{2}$. Manuscr. Math. 77, 415-433 (1992)

[CheL] Chen, W., Li, C.: Classification of solutions of some nonlinear elliptic equations. Duke Math. J. 63, 615-622 (1991)

[CY] Chang, S.-Y.A., Yang, P.C.: Prescribing Gaussian curvature on $S^{2}$. Acta Math. 159, 215-259 (1987)

[ES] Eyink, G., Spohn, H.: Negative temperature states and large-scale long-lived vortices in two-dimensional turbulence. J. Stat. Phys. 70, 833-886 (1993)

[GP] Garrett, A.J.M., Poladian, L.: Refined derivation, exact solutions and singular limits of the Poisson Boltzmann equation. Ann. Phys. (N.Y.) 188, 386-435 (1988)

[GNN1] Gidas, B., Ni, W.M., Nirenberg, L.: Symmetry and related properties via the maximum principle. Commun. Math. Phys. 68, 209-243 (1979)

[GNN2] Gidas, B., Ni, W.M., Nirenberg, L.: Symmetry of positive solutions of non linear elliptic equations in $\mathbb{R}^{n}$. Math. Anal. and Appl. Part A, Adv. Math. Suppl. Studies 7A, Nachbin, L. (ed.), New York: Acad. Press, 1981, pp. 369-402

[GT] Gilbarg, D., Trudinger, N.: Elliptic partial differential equations of second order. New York: Springer Verlag 1983

[KW] Kazdan, J., Warner, F.: Curvature functions for compact 2-manifolds. Ann. Math. 99, 14-47 (1974)

[K] Kiessling, M.K.-H.: Statistical mechanics of classical particles with logarithmic interactions. Commun. Pure Appl. Math. 46, 27-56 (1993)

[Li] Li, C.-M.: Monotonicity and symmetry of solutions of fully nonlinear elliptic equations on unbounded domains. Commun. PDE 16, 585-615 (1991) 
[LiNi] Li, Y., Ni, W.M.: On the existence and symmetry properties of finite total mass solutions of the Matukama equation, the Eddington equation, and their generalizations. Arch. Rat. Mech. Anal. 108, 175-194 (1989)

[LiNi2] Li, Y., Ni, W.M.: On the asymptotic behavior and radial symmetry of positive solutions of semilinear elliptic equations in $\mathbb{R}^{n}$, I. Arch. Rat. Mech. Anal. 118, 195-222 (1992)

[LiNi3] Li, Y., Ni, W.M.: On the asymptotic behavior and radial symmetry of positive solutions of semilinear elliptic equations in $\mathbb{R}^{n}$, II. Arch. Rat. Mech. Anal. 118, 223-243 (1992)

[Lie] Lieb, E.H.: Sharp constants in the Hardy-Littlewood-Sobolev and related inequalities. Ann. Math. 118, 349-374 (1983)

[MMSMO] Montgomery, D., Matthaeus, W.H., Stribling, W.T., Martinez, D., Oughton, S.: Relaxation in two dimensions and the "sinh-Poisson" equation. Phys. Fluids A 4, 3-7 (1992)

[Ob] Obata, M.: The conjectures on conformal transformations of Riemannian manifolds. J. Diff. Geom. 6, 247-258 (1971)

[On] Onofri, E.: On the positivitiy of the effective action in a theory of random surfaces. Commun. Math. Phys. 86, 321-326 (1982)

[OV] Onofri, E., Virasoro, M.: On a formulation of Polyakov's string theory with regular classical solutions. Nucl. Phys. B 201, 159-175 (1982)

[Ons] Onsager, L.: Statistical hydrodynamics. Nuovo Cim. Suppl. 6, 279-287 (1949)

[OPS] Osgood, B., Phillips, R., Sarnak, P.: Extremals of determinants of Laplacians. J. Funct. Anal. 80, 148-211 (1988)

[PD] Polvani, L., Dritschel, D.: Wave and vortex dynamics on the surface of a sphere. J. Fluid Mech. 255, 35-64 (1993)

[SB] Schmid-Burgk, J.: Finite amplitude density variations in a self-gravitating isothermal gas layer. Astrophys. J. 149, 727-729 (1967)

[Sch] Schoen, R.: Conformal deformation of a Riemann metric to constant scalar curvature. J. Diff. Geom. 20, 479-495 (1985)

[SO'N] Smith, R.A., O'Neil, T: Nonaxisymmetric thermal equilibria of a cylindrically bounded guiding center plasma or discrete vortex system. Phys. Fluids B 2, 2961-2975 (1990)

[St] Stuart, J.T.: On finite amplitude oscillations in laminar mixing layers. J. Fluid Mech. 29, 417-439 (1967)

[Wilc] Wilczek, F.: Geometry and interaction of instantons. In: Stump, D., Weingarten, D. (eds.) Quark confinement and field theory. New York: Wiley 1977

[Will] Williamson, J.H.: Statistical mechanics of a guiding center plasma. J. Plasma Phys. 17, 85-92 (1977)

Communicated by J.L. Lebowitz 